\title{
Leibniz's Laws of Continuity and Homogeneity
}

\author{
Mikhail G. Katz and David M. Sherry
}

\section{From the Characteristica Universalis to Ideal Entities}

Leibniz envisioned the creation of a universal language, characteristica universalis, ambitiously designed to serve as the vehicle for deduction and discovery in all fields of knowledge. Couturat [10] pointed out in 1901 that, in Leibniz's vision, the infinitesimal calculus was but the first salvo, or sample, of his characteristica universalis.

Leibniz's vision of the ideal nature of mathematical entities was remarkably modern. His description of infinitesimals as fictional entities shocked his disciples J. Bernoulli, l'Hôpital, and Varignon. And his infinitesimals certainly appeared as "Mysteries" to critics such as Berkeley, whose empiricist philosophy tolerated no conceptual innovations, such as infinitesimals, without an empirical counterpart or referent:

Yet some there are, who, though they shrink at all other Mysteries, make no difficulty of their own, who strain at a Gnat and swallow a Camel [1, section XXXIV].

While today we are puzzled by Berkeley's rigid rejection of the idea of an infinitely divisible continuum, he also articulated a specifically logical criticism of the calculus (see [46]), alleging that

Mikhail G. Katz is professor of mathematics at Bar Ilan University. His email address is katzmik@macs. biu . ac. $i 1$.

David M. Sherry is professor of philosophy at Northern Arizona University. His email address is David. Sherry@ nau.edu.

DOI: http://dx.doi.org/10.1090/noti921 the system suffered from logical flaws and even contradictions. Even today, many historians believe Berkeley's criticism to have been on target. Not even Robinson escaped this trend, praising Berkeley's criticism of the foundations of the calculus as "a brilliant exposure of their logical inconsistencies" [45, p. 280].

We argue that, contrary to Berkeley's view, Leibniz's system for the differential calculus was robust and free of contradiction. Leibniz articulated a set of coherent heuristic procedures for his calculus. Thus, Leibniz's system incorporated versatile heuristic principles, such as his law of continuity and laws of homogeneity, which were amenable, in the ripeness of time, to implementation as general principles governing the manipulation of modern infinitesimal and infinitely large quantities, such as the transfer principle and the standard part principle. Kanovei [21] and others performed similar reconstructions of Euler's work.

We will draw on Leibniz's work, more specifically his Cum Prodiisset, to argue for the consistency of Leibniz's system for the differential calculus. ${ }^{1}$ We will also draw on the work of Leibniz historians Bos, Ferraro, Horváth, Knobloch, and Laugwitz.

Berkeley's attack on infinitesimal calculus focused specifically on the product rule as well as

\footnotetext{
${ }^{1}$ The text Cum Prodiisset sheds more light on foundational issues than the terser 1684 text Nova methodus pro maximis et minimis... [35], so named because the determination of maxima and minima was one of the central problems of analysis as practiced at the time, with leading scholars having composed works of similar titles (see, e.g., [12]).
} 
on the derivation of polynomials. Once the logical contradiction, alleged by Berkeley, is resolved in the context of the product rule (see the section "Justification of the Product Rule" below), it can similarly be resolved (namely, by applying the transcendental law of homogeneity) in all other contexts.

In a seminal 1974 study of Leibnizian methodology, Bos described a pair of distinct approaches to justifying the calculus:

Leibniz considered two different approaches to the foundations of the calculus; one connected with the classical methods of proof by "exhaustion", the other in connection with a law of continuity [5, item 4.2, p. 55].

The first approach relies on an Archimedean "exhaustion" methodology. We will therefore refer to it as the A-methodology. The other methodology exploits infinitesimals and the law of continuity. We will refer to it as the B-methodology, in an allusion to Johann Bernoulli, who, having learned an infinitesimal methodology from Leibniz, never wavered from it.

Knobloch explains the role of Leibniz's law of continuity in the following terms:

In his treatise Leibniz used a dozen rules which constitute his arithmetic of the infinite. He just applied them without demonstrating them, only relying on the law of continuity: The rule of the finite remains valid in the domain of the infinite [30, p. 67].

Laugwitz pointed out that Leibniz's law of continuity

contains an a priori assumption: our mathematical universe of discourse contains both finite objects and infinite ones [32, p. 145].

What is the ontological status of such infinitary (infinitesimal or infinite) objects in Leibniz's theory? Leibniz's was a remarkably modern insight that mathematical entities need not have a referent, or empirical counterpart. The fictional nature of infinitesimals was stressed by Leibniz in 1706:

Philosophically speaking, I no more admit magnitudes infinitely small than infinitely great...I take both for mental fictions, as more convenient ways of speaking, and adapted to calculation, just like imaginary roots are in algebra (Leibniz to Des Bosses, 11 March 1706, in [16, II, p. 305]).

Infinitesimals, like imaginaries, were wellfounded fictions to Leibniz. The nature of Leibniz's infinitesimals is further clarified by Ferraro, who analyzes a lengthy quotation from Leibniz's famous letter of 1702 [38] to Varignon (which we do not reproduce here to save space) in the following terms:
According to Leibniz, imaginary numbers, infinite numbers, infinitesimals, the powers whose exponents were not "ordinary" numbers and other mathematical notions are not mere inventions; they are auxiliary and ideal quantities that...serve to shorten the path of thought [13, p. 35].

In Ferraro's view, Leibniz's infinitesimals enjoy an ideal ontological status similar to that of the complex numbers, surd (irrational) exponents, and other ideal quantities.

In the next section we will examine Leibniz's foundational stance as expressed in his seminal text Cum Prodiisset.

\section{Cum Prodiisset}

Leibniz's text Cum Prodiisset [37] (translated by Child [9]) dates from around 1701 according to modern scholars. The text is of crucial importance in understanding Leibniz's foundational stance. We will analyze it in detail in this section.

\section{Law of Continuity, with Examples}

Leibniz formulates his law of continuity in the following terms:

Proposito quocunque transitu continuo in aliquem terminum desinente, liceat raciocinationem communem instituere, qua ultimus terminus comprehendatur [37, p. 40].

The passage can be translated as follows:

In any supposed continuous transition, ending in any terminus, it is permissible to institute a general reasoning, in which the final terminus may also be included.

We have deliberately avoided using the term limit in our translation. ${ }^{2}$ In fact, translating terminus as limit would involve a methodological error, because the term limit misleadingly suggests its modern technical meaning of a real-valued operation applied to sequences or functions.

In a similar vein, Bos notes that

the fundamental concepts of the Leibnizian infinitesimal calculus can best be understood as extrapolations to the actually infinite of concepts of the calculus of finite sequences. I use the term "extrapolation"

\footnotetext{
${ }^{2}$ This is consonant with Child's translation: "In any supposed transition, ending in any terminus, it is permissible to institute a general reasoning, in which the final terminus may also be included" [9, p. 147]. We have reinstated the adjective continuous modifying transition (deleted by Child possibly in an attempt to downplay a perceived logical circularity of defining a law of continuity in terms of continuity itself).
} 
here to preclude any idea of taking a limit $[5, \text { p. } 13]^{3}$

Leibniz gives several examples of the application of his law of continuity. We will focus on the following three examples.

(1) In the context of a discussion of parallel lines, he writes:

When the straight line BP ultimately becomes parallel to the straight line VA, even then it converges toward it or makes an angle with it, only that the angle is then infinitely small $[9$, p. 148].

(2) Invoking the idea that the term equality may refer to equality up to an infinitesimal error, Leibniz writes:

When one straight line ${ }^{4}$ is equal to another, it is said to be unequal to it, but that the difference is infinitely small [9, p. 148]. ${ }^{5}$

(3) Finally, a conception of a parabola expressed by means of an ellipse with an infinitely removed focal point is evoked in the following terms:

A parabola is the ultimate form of an ellipse, in which the second focus is at an infinite distance from the given focus nearest to the given vertex [9, p. 148].

\section{Status Transitus}

We return to our analysis of the law of continuity as formulated in Cum Prodiisset. Leibniz introduces his next observation by the clause "of course it is really true that" and notes that "straight lines which are parallel never meet" [9, p. 148], that "things which are absolutely equal have a difference which is absolutely nothing" [9, p. 148], and that "a parabola is not an ellipse at all" [9, p. 149]. These remarks would seem to rule out the wondrous entities of the previous subsection. How does one, then, account for these examples? Leibniz provides an explanation in terms of a state of transition (status transitus in the original Latin [37, p. 42]):

A state of transition may be imagined, or one of evanescence, in which indeed there has not yet arisen exact equality...or

\footnotetext{
${ }_{3}^{3}$ Bos goes on specifically to criticize the Bourbaki's limite wording "(Leibniz) se tient très près du calcul des différences, dont son calcul différentiel se déduit par un passage à la limite" [6, p. 208].

${ }^{4}$ Here Leibniz is using the term line in its generic meaning of a segment.

${ }^{5}$ Equality up to an infinitesimal is a state of transition from inequality to equality (this anticipates the transcendental law of homogeneity, dealt with in the section "Assignable and Inassignable Quantities" below).
}

parallelism, but in which it is passing into such a state, that the difference is less than any assignable quantity; also that in this state there will still remain some difference,... some angle, but in each case one that is infinitely small; and the distance of the point of intersection, or the variable focus, from the fixed focus will be infinitely great, and the parabola may be included under the heading of an ellipse [9, p. 149].

A state of transition in which "there has not yet arisen exact equality" refers to example (2) in the previous subsection; "parallelism" refers to example (1); including parabola under the heading of ellipse is example (3).

Thus, the term terminus encompasses the status transitus, involving a passage into an assignable entity while being as yet inassignable. Translating terminus as limit amounts to translating it as an assignable entity, the antonym of the meaning intended by Leibniz.

The observation that Leibniz's status transitus is an inassignable quantity is confirmed by Leibniz's conceding that its metaphysical status is "open to question":

Whether such a state of instantaneous transition from inequality to equality,...from convergence [i.e., lines meeting-] to parallelism, or anything of the sort, can be sustained in a rigorous or metaphysical sense, or whether infinite extensions successively greater and greater, or infinitely small ones successively less and less, are legitimate considerations, is a matter that I own to be possibly open to question [9, p. 149].

Yet Leibniz asserts that infinitesimals may be utilized independently of metaphysical controversies:

But for him who would discuss these matters, it is not necessary to fall back upon metaphysical controversies, such as the composition of the continuum, or to make geometrical matters depend thereon [9, pp. 149-150].

To summarize, Leibniz holds that the inassignable status of status transitus is no obstacle to its effective use in geometry. The point is reiterated in the next paragraph:

If any one wishes to understand these [i.e., the infinitely great or the infinitely small-] as the ultimate things, or as truly infinite, it can be done, and that too without falling back upon a controversy about the reality of extensions, or of infinite continuums in general, or of the infinitely small, ay, even though he think that such things 
are utterly impossible; it will be sufficient simply to make use of them as a tool that has advantages for the purpose of the calculation, just as the algebraists retain imaginary roots with great profit [9, p. 150].

Leibniz has just asserted the possibility of the mathematical infinite: "it can be done", without philosophical commitments as to its ontological reality.

\section{Mathematical Implementation of Status Transitus}

We will illustrate Leibniz's concept of status transitus by implementing it mathematically in the three examples mentioned by Leibniz.

In the subsection "Status Transitus", we mentioned that Leibniz viewed infinitesimals as fictions, and so his methods avoided any metaphysical commitments. Ishiguro [20] and others viewed a Leibnizian infinitesimal as a logical fiction, involving a syncategorematic paraphrase with a hidden quantifier applied to ordinary real values. We have argued against the syncategorematic interpretation in [28]. Rather, Leibnizian infinitesimals are pure fictions akin to imaginaries. Thus, Leibniz exploited infinitely large and infinitely small quantities in the same sense in which Albert Girard (1595-1632) and others exploited imaginary roots in order to simplify algebra. In neither case is there a commitment to corresponding mathematical entities. Thus, Leibniz anticipates modern formalist positions such as Hilbert's and Robinson's.

Of course, the structural properties of Leibniz's infinite and infinitely small quantities are different from those of modern day infinitesimals. Nonetheless, modern theories of infinitesimals are a way of implementing Leibniz's heuristic procedures. Thus, example (2) can be illustrated as follows. Leibniz denotes a finite positive quantity by

$$
\text { (d) } x
$$

(Bos [5, p. 57] replaced this by $\underline{\mathrm{d} x}$ ). The assignable quantity $(d) x$ passes via infinitesimal $d x$ on its way to absolute 0 . Then the infinitesimal $d x$ is the status transitus. Zero is merely the assignable shadow ${ }^{6}$ of the infinitesimal. Then a line (i.e., segment) of length $2 x+d x$ will be equal to one of length $2 x$, up to an infinitesimal. This particular status transitus is the foundation of the Leibnizian definition of the differential quotient.

Example (1) of parallel lines can be elaborated as follows. Let us follow Leibniz in building the line through $(0,1)$ parallel to the $x$-axis in the plane. Line $L_{H}$ with $y$-intercept 1 and $x$-intercept $H$ is given by $y=1-\frac{x}{H}$. For infinite $H$, the line $L_{H}$ has negative infinitesimal slope, meets the $x$-axis at an

\footnotetext{
${ }^{6}$ See footnote 7 .
}

infinite point, and forms an infinitesimal angle with the $x$-axis at the point where they meet. We will denote by st $(x)$ the assignable (i.e., real) shadow ${ }^{7}$ of a finite $x$. Then every finite point $(x, y) \in L_{H}$ satisfies

$$
\begin{aligned}
\operatorname{st}(x, y) & =(\operatorname{st}(x), \operatorname{st}(y)) \\
& =\left(\operatorname{st}(x), \operatorname{st}\left(1-\frac{x}{H}\right)\right) \\
& =(\operatorname{st}(x), 1) .
\end{aligned}
$$

Hence the finite portion of $L_{H}$ is infinitely close to the line $y=1$ parallel to the $x$-axis, which is its shadow. Thus, the parallel line is constructed by varying the oblique line depending on a parameter. Such variation passes via the status transitus defined by an infinite value of $H$.

To implement example (3), let's follow Leibniz in deforming an ellipse, via a status transitus, into a parabola. The ellipse with vertex (apex) at $(0,-1)$ and with foci at the origin and at $(0 ; H)$ is given by

$$
\sqrt{x^{2}+y^{2}}+\sqrt{x^{2}+(y-H)^{2}}=H+2 .
$$

We square (1) to obtain

$$
\begin{aligned}
x^{2}+ & y^{2}+x^{2}+(H-y)^{2} \\
& +2 \sqrt{\left(x^{2}+y^{2}\right)\left(x^{2}+(H-y)^{2}\right)} \\
= & H^{2}+4 H+4 .
\end{aligned}
$$

We move the radical to one side:

$$
\begin{aligned}
& 2 \sqrt{\left(x^{2}+y^{2}\right)\left(x^{2}+(H-y)^{2}\right)} \\
& \quad=H^{2}+4 H+4-\left(x^{2}+y^{2}+x^{2}+(H-y)^{2}\right)
\end{aligned}
$$

and square again. After cancellation we see that (1) is equivalent to

$$
\text { (4) }\left(y+2+\frac{2}{H}\right)^{2}-\left(x^{2}+y^{2}\right)\left(1+\frac{4}{H}+\frac{4}{H^{2}}\right)=0 \text {. }
$$

The calculation (1) through (4) depends on habits of general reasoning, such as:

- squaring undoes a radical,

- the binomial formula,

- terms in an equation can be transferred to the other side, etc.

General reasoning of this type is familiar in the realm of ordinary assignable (finite) numbers, but why does it remain valid when applied to the fictional "realm" of inassignable (infinite or infinitesimal) numbers? The validity of transferring such general reasoning, originally instituted in

\footnotetext{
${ }^{7}$ Here "st" stands for the standard part function in the context of the hyperreals. Of course, Leibniz used neither the term "shadow" nor the "st" notation. Rather, these notations from modern infinitesimal analysis implement mathematically a heuristic principle of Leibniz's called the transcendental law of homogeneity, discussed in the section "Assignable and Inassignable Quantities" below.
} 
the finite realm, to the "realm" of the infinite is precisely the content of Leibniz's law of continuity. ${ }^{8}$

We therefore apply Leibniz's law of continuity to equation (4) for an infinite $H$. The resulting entity is still an ellipse of sorts, to the extent that it satisfies all of the equations (1) through (4). However, this entity is no longer finite. It represents a Leibnizian status transitus between ellipse and parabola. This status transitus has foci at the origin and at an infinitely distant point $(0, H)$. Assuming $x$ and $y$ are finite, we set $x_{0}=\operatorname{st}(x)$ and $y_{0}=\operatorname{st}(y)$ to obtain an equation for a real shadow of this entity:

$$
\begin{aligned}
\text { st } & \left(\left(y+2+\frac{2}{H}\right)^{2}-\left(x^{2}+y^{2}\right)\left(1+\frac{4}{H}+\frac{4}{H^{2}}\right)\right) \\
= & \left(y_{0}+2+\operatorname{st}\left(\frac{2}{H}\right)\right)^{2} \\
& -\left(x_{0}^{2}+y_{0}^{2}\right)\left(1+\operatorname{st}\left(\frac{4}{H}+\frac{4}{H^{2}}\right)\right) \\
= & \left(y_{0}+2\right)^{2}-\left(x_{0}^{2}+y_{0}^{2}\right) \\
= & 0 .
\end{aligned}
$$

Simplifying, we obtain

$$
y_{0}=\frac{x_{0}^{2}}{4}-1 .
$$

Thus, the finite portion of the status transitus (4) is infinitely close to its shadow (5), namely, the real parabola $y=\frac{x^{2}}{4}-1$. This is the kind of payoff Leibniz is seeking with his law of continuity.

Some historians have been reluctant to interpret Leibniz's mathematics in terms of modern mathematical theories. Thus, Dauben presents a list of authors, including Detlef Laugwitz, who "have used nonstandard analysis to rehabilitate or 'vindicate' earlier infinitesimalists," and concludes:

Leibniz, Euler, and Cauchy...had, in the views of some commentators, "Robinsonian" nonstandard infinitesimals in mind from the beginning. The most detailed and methodically [sic] sophisticated of such treatments to date is that provided by Imre Lakatos; in what follows, it is his analysis of Cauchy that is emphasized [11, p. 179].

However, Lakatos's treatment was certainly not "the most detailed and methodically sophisticated" one by the time Dauben's text appeared in 1988. Thus, in 1987, Laugwitz had published a detailed scholarly study of Cauchy in Historia Mathematica [31]. Laugwitz's text in Historia Mathematica seems to be the published version of his 1985 preprint "Cauchy and infinitesimals". Laugwitz's 1985 preprint does appear in Dauben's bibliography [11, p. 199], indicating that Dauben was familiar with it. It is odd to suggest, as Dauben

\footnotetext{
${ }^{8}$ When the general reasoning being transferred to the infinite "realm" is generalized to encompass arbitrary elementary properties (i.e., first-order properties), one obtains the Łoś-Robinson transfer principle.
}

seems to, that a scholarly study published in Historia Mathematica would countenance a view that Leibniz and Cauchy could have had "'Robinsonian' nonstandard infinitesimals in mind from the beginning." Surely Dauben has committed a strawman fallacy here.

To a historian, the claim that Leibniz's differential calculus was free of logical fallacies may seem analogous to claiming that the circle can be squared, ${ }^{9}$ but only if the historian embraces the triumviratist story of analysis as an ineluctable march from incoherent infinitesimalism toward the yawning heights of Weierstrassian epsilontics.

Rather, Lakatos, Laugwitz, Bråting [8], and others have argued that infinitesimals as employed by Leibniz, Euler, and Cauchy have found a settheoretic implementation in the framework of modern theories of infinitesimals. The existence of such implementations indicates that the historical infinitesimals were less prone to contradiction than has been routinely maintained by triumvirate historians. ${ }^{10}$ The issue is dealt with in more detail by Katz and Katz [22], [23], [24], [25]; Błaszczyk et al. [2]; Borovik et al. [3]; Katz and Leichtnam [26]; Katz, Schaps, and Shnider [27].

\section{Assignable versus Unassignable}

In this section we will retain the term "unassignable" from Child's translation [9] (inassignabiles in the original Latin; see [37, p. 46]). After introducing finite quantities $(d) x,(d) y,(d) z$, Leibniz notes that

the unassignables $d x$ and $d y$ may be substi-

tuted for them by a method of supposition

even in the case when they are evanescent [9, p. 153].

Leibniz proceeds to derive his multiplicative law in the case $a y=x v$. Simplifying the differential quotient, Leibniz obtains

$$
\frac{a d y}{d x}=\frac{x d v}{d x}+v+d v
$$

At this point Leibniz proposes to transfer "the matter, as we may, to straight lines that never become evanescent," obtaining ${ }^{11}$

$$
\frac{a(d) y}{(d) x}=\frac{x(d) v}{(d) x}+v+d v
$$

The advantage of (7) over (6) is that the expressions $\frac{(d) y}{(d) x}$ and $\frac{(d) v}{(d) x}$ are assignable (real). At this stage, Leibniz points out that " $d v$ is superfluous." The reason given is that "it alone can become evanescent."

\footnotetext{
${ }^{9}$ Such was indeed the tenor of a recent referee report; see http://u.cs.biu.ac.i1/ katzmik/straw2.htm7

${ }^{10}$ C. Boyer refers to Cantor, Dedekind, and Weierstrass as "the great triumvirate;" see ([7, p. 298]).

${ }^{11}$ Child incorrectly transcribes formula (7) from Gerhardt, replacing the equality sign in Gerhardt by a plus sign. Note that Leibniz himself used the sign $\because$ (see [42, p. 371]).
} 
The transcendental law of homogeneity (see the section "Assignable and Inassignable Quantities") is not mentioned explicitly in Cum Prodiisset; therefore the discussion of this step necessarily remains a bit vague. Discarding the $d v$ term, one obtains the expected product formula $\frac{a(d) y}{(d) x}=\frac{x(d) v}{(d) x}+v$ in this case. Note that thinking of the left-hand side of (7) as the assignable shadow of the right-hand side is consistent with Leibniz's example (2) (see the subsection "Law of Continuity, with Examples").

\section{Souverain Principe}

In a February 2, 1702, letter to Varignon, Leibniz formulated the law of continuity as follows:

...et il se trouve que les règles du fini réussissent dans l'infini comme s'il y avait des atomes (c'est à dire des éléments assignables de la nature) quoiqu'il n'y en ait point la matière étant actuellement sousdivisée sans fin; et que vice versa les règles de l'infini réussissent dans le fini, comme s'il y'avait des infiniment petits métaphysiques, quoiqu'on n'en n'ait point besoin; et que la division de la matière ne parvienne jamais à des parcelles infiniment petites: c'est parce que tout se gouverne par raison, et qu'autrement il n'aurait point de science ni règle, ce qui ne serait point conforme avec la nature du souverain principe [38, p. 350].

This formulation was cited by Robinson in 1966, [45, p. 262]. To summarize: the rules of the finite succeed in the infinite, and conversely.

\section{Assignable and Inassignable Quantities}

How did Leibniz view the relation of assignable and inassignable quantities?

\section{Relation of Being Infinitely Close}

The rule governing infinitesimal calculation that Knobloch represents as Leibniz's rule 2.2 states:

$$
\begin{aligned}
& \text { 2.2. } x, y \text { finite, } x=(y+\text { infinitely small }) \Longleftrightarrow \\
& x-y \approx 0 \text { (not assignable difference) [30, } \\
& \text { p. 67]. }
\end{aligned}
$$

Here the pair of parallel wavy lines represents the relation of being infinitely close. Leibnizian assignable quantities mark locations in what would be called today an Archimedean continuum, or A-continuum for short. Such a continuum stems from the sixteenth-century work of Simon Stevin (1548-1620) [47], [48]. Stevin initiated a systematic approach to decimal representation of measuring numbers, marking a transition from a discrete arithmetic as practiced by the Greeks, to the arithmetic of the continuum taken for granted today (see [41], [44], and [2]).
Closely related to the distinction between the A- and B-methodologies is a distinction between two types of continua, which could be called an A-continuum and a B-continuum. The latter encompasses inassignable entities such as infinitesimals (in addition to assignable ones) and can be described as a "thick" continuum. ${ }^{12}$ On occasion, Leibniz describes such entities as "incomparable quantities" and defines them in terms of the violation of what today is called the Archimedean property. Thus, Leibniz writes in a letter to l'Hôpital:

I call incomparable quantities of which the one can not become larger than the other if multiplied by any finite number. This conception is in accordance with the fifth definition of the fifth book of Euclid ([36, p. 288], cited in [19, p. 63]). ${ }^{13}$

\section{Transcendental Law of Homogeneity}

To mediate between assignable and inassignable quantities, Leibniz developed an additional principle called the transcendental law of homogeneity. Leibniz's transcendental law of homogeneity governs equations involving differentials. Bos interprets it as follows:

A quantity which is infinitely small with respect to another quantity can be neglected if compared with that quantity. Thus all terms in an equation except those of the highest order of infinity, or the lowest order of infinite smallness, can be discarded. For instance,

(8)

$$
\begin{gathered}
a+d x=a, \\
d x+d d y=d x,
\end{gathered}
$$

etc. The resulting equations satisfy this...requirement of homogeneity [5, p. 33], paraphrasing [39, pp. 381-382].

The title of Leibniz's 1710 text is Symbolismus memorabilis calculi algebraici et infinitesimalis in comparatione potentiarum et differentiarum, et de lege homogeneorum transcendentali. The inclusion of the transcendental law of homogeneity (lex homogeneorum transcendentalis) in the title of the text attests to the importance Leibniz attached to this law.

How did Leibniz use the transcendental law of homogeneity in developing the calculus? In the next section we will illustrate an application of the transcendental law of homogeneity to the particular example of the derivation of the product rule.

\footnotetext{
${ }^{12}$ The B-continuum can be thought of as "thicker" than the A-continuum, because the B-continuum is, as it were, packed chock-full of numbers, including infinitesimals.

${ }^{13}$ Horváth notes that Leibniz is actually referring to the fourth definition of the fifth book.
} 


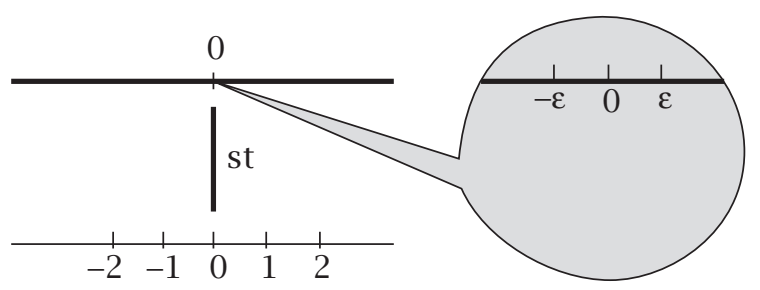

Figure 1. Zooming in on infinitesimal $\epsilon$ (here $\operatorname{st}( \pm \epsilon)=0)$.

\section{Justification of the Product Rule}

The issue is the justification of the last step in the following calculation:

$$
\begin{aligned}
d(u v) & =(u+d u)(v+d v)-u v \\
& =u d v+v d u+d u d v \\
& =u d v+v d u .
\end{aligned}
$$

The last step in the calculation (9), namely,

$$
u d v+v d u+d u d v=u d v+v d u,
$$

is an application of Leibniz's law of homogeneity. ${ }^{14}$

In his 1701 text Cum Prodiisset [37, pp. 46-47], Leibniz presents an alternative justification of the product rule (see [5, p. 58]). Here he divides by $d x$ and argues with differential quotients rather than differentials. We analyzed Leibniz's calculation in the subsection "Assignable versus Unassignable". Adjusting Leibniz's notation to fit with (9), we obtain an equivalent calculation: ${ }^{15}$

$$
\begin{aligned}
\frac{d(u v)}{d x} & =\frac{(u+d u)(v+d v)-u v}{d x} \\
& =\frac{u d v+v d u+d u d v}{d x} \\
& =\frac{u d v+v d u}{d x}+\frac{d u d v}{d x} \\
& =\frac{u d v+v d u}{d x} .
\end{aligned}
$$

Under suitable conditions the term $\frac{d u d v}{d x}$ is infinitesimal, and therefore the last step

$$
\frac{u d v+v d u}{d x}+\frac{d u d v}{d x}=u \frac{d v}{d x}+v \frac{d u}{d x}
$$

is legitimized as a special case of the transcendental law of homogeneity, which interprets the equality sign in (8) as the relation of being infinitely close, i.e., an equality up to infinitesimal error. Note that the use of the equality sign "=" to denote a nonsymmetric relation of discarding the "inhomogeneous" terms as in (8) and (9) should

\footnotetext{
${ }^{14}$ Leibniz had two laws of homogeneity, one for dimension and the other for the order of infinitesimalness. Bos notes that they "disappeared from later developments" [5, p. 35], referring to Euler and Lagrange.

${ }^{15}$ The special case treated by Leibniz is $u(x)=x$. This limitation does not affect the conceptual structure of the argument.
}

hardly shock the modern reader accustomed to the "big O" notation: we write $\sin x=O(1)$, but we would certainly not write $O(1)=\sin x$. Leibniz's transcendental law of homogeneity involved such an "asymmetric" relation, since it replaced an inassignable quantity such as $2 x+d x$ by the assignable quantity $2 x$.

\section{Was Leibniz's System for Differential Calculus Consistent?}

Berkeley's logical criticism of the calculus is that the evanescent increment is first assumed to be nonzero to set up an algebraic expression and then treated as zero in discarding the terms that contained that increment when the increment is said to vanish. The criticism, however, involves a misunderstanding of Leibniz's method. The rebuttal of Berkeley's criticism is that the evanescent increment need not be "treated as zero" but, rather, merely discarded through an application of the transcendental law of homogeneity by Leibniz, as illustrated in the previous section in the case of the product rule.

While consistent, Leibniz's system unquestionably relied on heuristic principles, such as the laws of continuity and homogeneity, and would thus fall short of a standard of rigor if measured by today's criteria. On the other hand, the consistency and resilience of Leibniz's system is confirmed through the development of modern implementations of Leibniz's heuristic principles. Thus, in the 1940s, Hewitt [18] developed a modern implementation of a hyperreal B-continuum extending $\mathbb{R}$ by means of a technique referred to today as the ultrapower construction. We will denote such a B-continuum by the new symbol $\mathbb{R}$. Denoting by $\mathbb{R}_{\infty}$ the subset of $\mathbb{R}$ consisting of inverses of nonzero infinitesimals, we obtain a partition $\mathbb{R}=\mathbb{R}_{<\infty} \cup \mathbb{R}_{\infty}$, where $\mathbb{R}_{<\infty}$ is the complement of $\mathbb{R}_{\infty}$. We then have the standard part function

$$
\text { st : } \mathbb{R}_{<\infty} \rightarrow \mathbb{R},
$$

illustrated in Figure 1. Note that the hyperreals can be constructed out of integers (see [4]). The traditional quotient construction using Cauchy sequences, usually attributed to Cantor (and actually due to Méray [43], who published three years earlier than E. Heine), can be factored through the hyperreals (see [17]). In 1955, Łoś [40] proved his celebrated theorem on ultraproducts, implying in particular that elementary (more generally, firstorder) statements over $\mathbb{R}$ are true if and only if they are true over $\mathbb{R}$, yielding a modern implementation of the Leibnizian law of continuity. Such a result is equivalent to what is known in the literature as the transfer principle (see [29]). The map that associates to every finite element of $\mathbb{R}$ the real number infinitely close to it is known in the literature as 
the standard part function (10) (alternatively, the shadow). Such a map is a mathematical implementation of the Leibnizian transcendental law of homogeneity.

\section{Acknowledgments}

The authors are grateful to S. Wenmackers for helpful comments. The influence of Hilton Kramer (1928-2012) is obvious.

\section{References}

[1] G. BERKELEY, The Analyst, a Discourse Addressed to an Infidel Mathematician, 1734.

[2] P. BŁaszczyK, M. KATZ, and D. Sherry, Ten misconceptions from the history of analysis and their debunking, Foundations of Science, 2012. See http://dx.doi.org/10.1007/s10699-012-9285-8 and/http://arxiv.org/abs/1202.4153.

[3] A. BoROVIK and M. KATZ, Who gave you the CauchyWeierstrass tale? The dual history of rigorous calculus, Foundations of Science 17 (2012), no. 3, 245-276. See http://dx.doi.org/10.1007/s10699-011-9235-x and http://arxiv.org/abs/1108.2885.

[4] A. BOROVIK, R. JIN, and M. KATZ, An integer construction of infinitesimals: Toward a theory of Eudoxus hyperreals, Notre Dame Journal of Formal Logic 53 (2012), no. 4 557-570.

[5] H. J. M. Bos, Differentials, higher-order differentials and the derivative in the Leibnizian calculus, Arch. History Exact Sci. 14 (1974), 1-90.

[6] N. BOURBAKI, Eléments d'histoire des mathématiques, Histoire de la Pensée. IV, Hermann, Paris, 1960.

[7] C. Boyer, The Concepts of the Calculus, Hafner Publishing Company, 1949.

[8] K. BRÅTING, A new look at E. G. Björling and the Cauchy sum theorem, Arch. Hist. Exact Sci. 61 (2007), no. 5, 519-535.

[9] CHILD, J. M. (ed.), The Early Mathematical Manuscripts of Leibniz, translated from the Latin texts published by Carl Immanuel Gerhardt, with critical and historical notes by J. M. Child, The Open Court Publishing Co., Chicago and London, 1920.

[10] L. Couturat, La logique de Leibniz, F. Alcan, Paris, 1901; reprinted, Olms, Hildesheim, 1961.

[11] J. DAUBEN, Abraham Robinson and nonstandard analysis: History, philosophy, and foundations of mathematics. In William Aspray and Philip Kitcher, eds., History and Philosophy of Modern Mathematics (Minneapolis, MN, 1985), pp. 177-200, Minnesota Stud. Philos. Sci., XI, Univ. of Minnesota Press, Minneapolis, MN, 1988. Available online at http://www.mcps.umn. edu/philosophy/ 11_7Dauben.pdf.

[12] P. FERMAT, Méthode pour la recherche du maximum et du minimum, pp. 121-156 in Tannery's edition [48].

[13] G. FERraro, Geometrical quantities and series in Leibniz. In The Rise and Development of the Theory of Series Up to the Early 1820s. Sources and Studies in the History of Mathematics and Physical Sciences, 2008, Part I, pp. 25-44. See http://dx.doi .org/10.1007/ 978-0-387-73468-2_2

[14] C. I. Gerhardt (ed.), Historia et Origo Calculi Differentialis a G. G. Leibnitio Conscripta, Hannover, 1846.
[15] C. I. Gerhardt (ed.), Leibnizens Mathematische Schriften, Eidmann, Berlin and Halle, 1850-1863.

[16] C. I. Gerhardt (ed.), G. W. Leibniz: Philosophische Schriften, 7 vols. (1875-1890). Reprint, Georg Olms Verlag, Hildesheim, 1962.

[17] P. GIORDANO and M. KATZ, Two ways of obtaining infinitesimals by refining Cantor's completion of the reals, preprint, 2011; see http://arxiv.org/abs/ 1109.3553

[18] E. HEWITT, Rings of real-valued continuous functions. I, Trans. Amer. Math. Soc. 64 (1948), 45-99.

[19] M. HoRvÁTH, On the attempts made by Leibniz to justify his calculus, Studia Leibnitiana 18 (1986), no. 1, 60-71.

[20] H. Ishiguro, Leibniz's Philosophy of Logic and Language, second edition, Cambridge University Press, Cambridge, 1990.

[21] V. KANOVEI, Correctness of the Euler method of decomposing the sine function into an infinite product (Russian), Uspekhi Mat. Nauk 43 (1988), no. 4 (262), 57-81, 255; translation in Russian Math. Surveys 43 (1988), no. 4, 65-94.

[22] K. KATZ and M. KATZ, A Burgessian critique of nominalistic tendencies in contemporary mathematics and its historiography, Foundations of Science 17 (2012), no. 1, 51-89. See http://dx.doi.org/ 10.1007/s10699-011-9223-1 and http://arxiv. org/abs/1104.0375.

[23] , Cauchy's continuum, Perspectives on Science 19 (2011), no. 4, 426-452. See http:// arxiv.org/abs/1108.4201 and http://www. mitpressiournals.org/doi/abs/10.1162/ POSC_a_00047

[24] , Stevin numbers and reality, Foundations of Science, 17 (2012), no. 2, 109-123. See http://arxiv.org/abs/1107.3688 and http:// dx.doi.org/10.1007/s10699-011-9228-9.

[25] , Meaning in classical mathematics: Is it at odds with intuitionism? Intellectica 56 (2011), no. 2, 223302. See http://arxiv.org/abs/1110.5456,

[26] M. Katz and E. LeICHTNAM, Commuting and noncommuting infinitesimals, American Mathematical Monthly (to appear).

[27] M. KATZ, D. SCHAPS, and S. SHNIDER, Almost equal: The method of adequality from Diophantus to Fermat and beyond, Perspectives on Science 20 (2012), no. 4, to appear.

[28] M. KATZ and D. SHERRY, Leibniz's infinitesimals: Their fictionality, their modern implementations, and their foes from Berkeley to Russell and beyond, Erkenntnis, 2012 (online first). See http:// dx.doi.org/10.1007/s10670-012-9370-y and http://arxiv.org/abs/1205.0174.

[29] H. J. KeISLER, The ultraproduct construction, Proceedings of the Ultramath Conference, Pisa, Italy, 2008, Contemp. Math., 530, Amer. Math. Soc., Providence, RI, 2010.

[30] E. KNOBLOCH, Leibniz's rigorous foundation of infinitesimal geometry by means of Riemannian sums. Foundations of the formal sciences I (Berlin, 1999), Synthese 133 (2002), no. 1-2, 59-73.

[31] D. LAugwiTz, Infinitely small quantities in Cauchy's textbooks, Historia Math. 14 (1987), no. 3, 258-274.

[32] __ Leibniz' principle and omega calculus, Le labyrinthe du continu (Colloq., Cerisy-la-Salle, France, 1990), Springer, Paris, 1992, pp. 144-154. 


\section{About the Cover}

\section{Does it get any better?}

The theme of this month's cover is taken from the article by Jeff Lagarias and Chuanming Zong in this issue. As they mention, the highest known packing density of regular tetrahedra is $4000 / 4671$ (about $0.856347 \ldots$..). The packing is reported in "Dense crystalline dimer packings of regular tetrahedra", an article in Discrete and Computational Geometry by Elizabeth Chen, Michael Engel, and Sharon Glotzer. It is a lattice made up of a basic assembly of 4 regular tetrahedra, grouped in pairs. Each pair, called a dimer, is a pair of tetrahedra joining face-toface. One of these dimers is the inversion of the other, so that the two dimers are complementary, and are tightly bound to each other, with faces intersecting as in the figure at left on the cover.

In the packing, a layer of dimers is laid out in a slightly rising plane. A layer of inverted dimers is then inserted tightly onto that one, and then more alternating layers are added. Some idea of how the layers fit can be seen in the top view on the cover, and in the figure below.

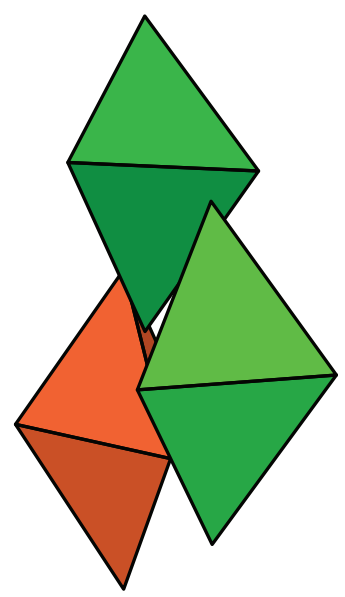

One thing notable about the packing is how similar it appears to the well-known packing of spheres proved not so long ago by Tom Hales to be optimal.

The packing was constructed so as to be optimal among lattice packings with faces intersecting tightly. Chen et al. include in their article some heuristic reasoning that they might in fact have discovered the densest possible packing of regular tetrahedra, although it is hard to imagine that this can ever be proved. The article includes in an appendix a remarkable set of pictures illustrating the construction, drawn by Chen.

$$
\begin{array}{r}
\text {-Bill Casselman } \\
\text { Graphics Editor } \\
\text { (notices-covers@ams.org) }
\end{array}
$$

[33] G. W. LeIBNIZ, De quadratura arithmetica circuli ellipseos et hyperbolae cujus corollarium est trigonometria sine tabulis, Kritisch herausgegeben und kommentiert von Eberhard Knobloch, Vandenboeck \& Ruprecht, Göttingen, 1993 [Abhandlungen der Akademie der Wissenschaften in Göttingen, Mathematisch-physikalische Klasse 3, 43].

[34] _ Quadrature Arithmétique du Cercle, de L'ellipse et de L'hyperbole, Marc Parmentier (trans. and ed.)/Latin text, Eberhard Knobloch (ed.), J. Vrin, Paris, 2004.

[35] _ Nova methodus pro maximis et minimis..., in Acta Erud., Oct. 1684. See [15], V, pp. 220-226.

[36] Leibniz to l'Hospital, 21 June 1695, in [15], II, pp. 287289.

[37] G. W. LeIBNIZ (1701) Cum Prodiisset..., "Cum prodiisset atque increbuisset Analysis mea infinitesimalis...", in [14], pp. 39-50.

[38] Leibniz to Varignon, 2 February, 1702, in [15], IV, pp. 91-95.

[39] G. W. LeIBNIZ, (1710) Symbolismus memorabilis calculi algebraici et infinitesimalis in comparatione potentiarum et differentiarum, et de lege homogeneorum transcendentali. In [15], V, pp. 377-382].

[40] J. Łoś, Quelques remarques, théorèmes et problèmes sur les classes définissables d'algèbres. In Mathematical Interpretation of Formal Systems, North-Holland Publishing Co., Amsterdam, 1955, pp. 98-113.

[41] A. MALET, Renaissance notions of number and magnitude, Historia Mathematica 33 (2006), no. 1, 63-81.

[42] R. B. MCCLENON, A contribution of Leibniz to the history of complex numbers. American Mathematical Monthly 30 (1923), no. 7, 369-374.

[43] H. C. R. MÉRAY, Remarques sur la nature des quantités définies par la condition de servir de limites à des variables données, Revue des sociétiés savantes des départments, Section sciences mathématiques, physiques et naturelles, 4th ser. 10 (1869), 280-289.

[44] J. NAETS, How to define a number? A general epistemological account of Simon Stevin's art of defining, Topoi 29 (2010), no. 1, 77-86.

[45] A. RobInson, Non-standard Analysis, North-Holland Publishing Co., Amsterdam, 1966.

[46] D. SHERRY, The wake of Berkeley's Analyst: rigor mathematicae? Stud. Hist. Philos. Sci. 18 (1987), no. 4, $455-480$

[47] S. STEVIN (1585), L'Arithmetique. In A. Girard (ed.), Les Oeuvres Mathematiques de Simon Stevin, Leyde, 1634, part I, pp. 1-101.

[48] , The Principal Works of Simon Stevin. Vols. IIA IIB: Mathematics, edited by D. J. Struik, C. V. Swets \& Zeitlinger, Amsterdam, 1958. Vol. IIA: v+pp. 1-455 (1 plate); Vol. IIB: iv+pp. 459-976.

[49] P. TANnery and C. Henry, Oeuvres de Fermat, Vol. 3, Gauthier-Villars, 1891. 